\title{
Regards croisés sur le secteur éditorial du théâtre contemporain jeunesse en France et en Europe
}

The Youth Theater Tublishing Sector in France and in Europe

\section{Pierre Banos-Ruf}

\section{OpenEdition}

\section{Journals}

Édition électronique

URL : https://journals.openedition.org/recherchestravaux/781

DOI : 10.4000/recherchestravaux.781

ISSN : 1969-6434

Éditeur

UGA Éditions/Université Grenoble Alpes

Édition imprimée

Date de publication : 1 décembre 2015

Pagination : 73-83

ISBN : 978-2-84310-314-8

ISSN : 0151-1874

\section{Référence électronique}

Pierre Banos-Ruf, « Regards croisés sur le secteur éditorial du théâtre contemporain jeunesse en

France et en Europe », Recherches \& Travaux [En ligne], 87 | 2015, mis en ligne le 01 janvier 2017, consulté le 29 octobre 2021. URL : http://journals.openedition.org/recherchestravaux/781 ; DOI : https://doi.org/10.4000/recherchestravaux.781 
Pierre Banos-RuF

Université Paris Ouest Nanterre

Éditions Théâtrales

\section{Regards croisés sur le secteur éditorial du théâtre contemporain jeunesse en France et en Europe}

Le texte de théâtre et son auteur, jadis éléments centraux du processus théâtral, ont été décentrés suite au «renversement copernicien ${ }^{\mathrm{I}}$ » du XIX ${ }^{\mathrm{e}}$ siècle au profit des metteurs en scène. Et si, depuis ces temps anciens, le texte a pu résister au scénocentrisme par des retours en grâce du fait de grands noms de la dramaturgie européenne, il n'en demeure pas moins un objet hybride engoncé entre littérature et spectacle, notamment quand on parle d'édition. Comme j'ai essayé de le démontrer dans ma thèse $e^{2}$, et depuis 2002 par mon travail d'éditeur' ${ }^{3}$, publier du théâtre est à la fois un acte militant, du fait d'une potentialité de lecture intrinsèque toute marginale, mais cela constitue une obligation culturelle pour ne pas trop perdre de cette matière vivante qui est à la fois partition et magma littéraire. Publier le théâtre est donc, tout à la fois, autoriser une trace, favoriser une source et fournir son écot à l'histoire littéraire. C'est particulièrement vrai depuis les années I980 en France où s'est agrégé un secteur spécialisé, une niche éditoriale constituée d'une vingtaine de structures uniquement dédiées à la littérature théâtrale (à condition d'inclure dans ce panel de spécialistes à la fois ce grand généraliste qu’est Actes Sud,

I. L'expression est de B. Dort dans un article de la revue Théâtres en I969.

2. L'édition théâtrale aujourd'hui. Enjeux politiques, économiques et esthétiques, thèse de doctorat sous la direction de C. Biet, université Paris Ouest Nanterre La Défense, 2008.

3. Directeur des Éditions Théâtrales depuis 20 II. 
leader du marché grâce à sa collection "Actes Sud-Papiers", qui accueille au sein d'un catalogue varié des textes de théâtre publiés depuis 1985 par une éditrice spécialisée, Claire David; et également L'École des loisirs, éditeur spécialisé en littérature jeunesse). Mais cette niche, qui à la fois protège et tend à confiner au ghetto, est laissée à la marge de l'édition industrielle, un ban d'où les éditeurs de théâtre sortent peu à peu, par quelques brevets de respectabilité littéraire ${ }^{4}$, et par un mouvement lancé vingt ans auparavant par quelques pionniers : l'édition de théâtre jeunesses.

Coincé donc entre littérature et spectacle, entre industrie et artisanat, cette niche de la publication du théâtre jeunesse se situe elle aussi entre théâtre et jeunesse. Pourtant, ce mouvement induit également une question de légitimité à acquérir pour l'édition de théâtre. Et cette diversification éditoriale en direction des textes dédiés, ou abordables par tout le champ de la jeunesse (de l'assemblée théâtrale - constituée des lieux, des compagnies, des ateliers -, au public plus pédagogique, jusqu'aux jeunes lecteurs et praticiens), a recouvert le secteur d'une légitimité plus importante encore du fait d'un accompagnement décisif du secteur à «l'émergence d'un répertoire ${ }^{6}$.

Car si les premières publications "spécialisées" de théâtre jeunesse apparaissent dans les années 1990 - j'exclus à dessein des publications plus anciennes mais dans une autre démarche - elles sont aussi issues du secteur naissant de «l'édition théâtrale» constitué ainsi dans les années I980, domaine éditorial alors moribond car abandonné par les grandes maisons de littérature comme l'a démontré Michel Vinaver dans Le compte rendu d'Avignon. Des mille maux dont souffre l'édition théatrale et des trente-sept remèdes pour l'en soulager (Actes Sud, 1987). Dans cet ouvrage, l'auteur dramatique montrait combien l'édition représentait au sein de l'assemblée théâtrale un maillon secondaire, voire pouvait être taxé de parasitisme. De façon plus optimiste, il pointait également les notions d'engagement et de militantisme nécessaires à ce sacerdoce peu rémunérateur. Et si la question se pose encore aujourd'hui, il importe de dépasser une posture corporatiste pour réfléchir ici, au-delà des thématiques et des esthétiques défendues par les éditeurs publiant du théâtre jeunesse, à l'enjeu de la transmission de ces ouvres, cela afin de pointer le rôle de l'édition dans sa découverte, ou comme l'écrit Michel Corvin pour le théâtre généraliste - le théâtre "tout public» - comment on passe d'un "théâtre du texte au

4. Le dramaturge L. Gaudé, découvert par C. David chez Actes Sud, offre par exemple le prix Goncourt à sa maison d'édition en 2004.

5. J'utilise l'expression de "théâtre jeunesse», plutôt que "théâtre jeune public», terminologie défendue par M. Bernanoce dans ses articles et ses ouvrages, qui me convient parfaitement pour défendre la potentialité de la lecture des textes de théâtre.

6. Ce terme provient du titre du n ${ }^{\circ} 9$ de la revue du SCÉRÉN, Théâtre aujourd'hui. 
théâtre de texte ${ }^{7}$. De plus, je chercherai à dépasser le seul marché français pour comprendre les ressorts de la publication des textes de théâtre jeunesse en Europe, car ceux-ci sont différents de la situation française.

\section{Quelques repères sur la constitution du secteur}

Afin d'observer la réalité du secteur de la publication du théâtre jeunesse, il est nécessaire de dresser un panorama rapide du secteur de l'édition de théâtre généraliste au sein duquel s'intègre notre objet. J'ai ainsi mis en évidence dans mes travaux trois marqueurs éditoriaux à partir desquels les éditeurs se déterminent pour définir leurs lignes : le « répertoire», le «théâtre comme littérature» et le «rapport à l'actualité théâtrale». Par ailleurs, le poids économique de cette niche du théâtre contemporain est d'une douzaine de millions d'euros (quand la littérature générale pèse 550 millions d'euros et l'édition française, 2,4 milliards d'euros). Environ 450 nouveautés sont publiées annuellement par $60 \%$ d'auteurs français, I2 \% d'auteurs francophones et $28 \%$ de traductions. Les deux tiers des titres publiés le sont par les éditeurs spécialisés et un tiers par des «éditeurs occasionnels» comme Gallimard, Minuit, P.O.L ou Le Seuil par exemple 8 .

Historiquement, le secteur du théâtre jeunesse connaît une trajectoire en partie semblable à celle du secteur généraliste, mais d'une façon "accélérée» : lien avec l'assemblée théâtrale plus qu'avec le monde du livre; émancipation progressive de l'actualité scénique immédiate; mise en livres soignée, mais discrète sur son appartenance au genre théâtral... Les premiers textes de théâtre jeunesse publiés, dans ce mouvement d'un répertoire contemporain dédié à la jeunesse, peuvent être datés de 1975 avec la collection des «Cahiers du Soleil debout", créée au sein du Théâtre des Jeunes Années de Lyon, dirigé par Maurice Yendt. Dans un premier temps, les pièces publiées accompagnent les créations du théâtre. Les années 1970 sont, comme on le sait, très importantes pour le secteur du théâtre jeune public, époque durant laquelle on assiste au passage des créations collectives issues du plateau à une certaine émancipation par quelques figures artistiques qui se muent en auteurs : Françoise Pillet (Théâtre de la Pomme Verte) ou Bruno Castan (Théâtre du Pélican), pour la

7. M. Corvin, La Lecture innombrable du texte de théatre contemporain, Paris, Éditions Théâtrales, 2015 .

8. Pour davantage de détails sur le secteur, je renvoie à divers articles en ligne, notamment : «L'édition théâtrale dans tous ses états» [en ligne], Les Lettres françaises, mai 2009. Disponible sur <http://www.les-lettres-francaises.fr/wp-content/uploads/20Io/II/2009I.pdf $>$ [consulté le I5/08/20I5]; "L'édition théâtrale à la croisée des chemins", Le Motif, juin 20IO, <http://www. lemotif.fr/fr/actualites/bdd/article/568>. 
France; Suzanne Lebeau (Théâtre du Carrousel) au Canada. Mais dix années sont nécessaires pour que l'aventure éditoriale devienne concrète pour ces auteurs et leurs textes.

Le véritable pionnier de la publication "autonome" des textes est Dominique Bérody qui crée en 1987 une collection de théâtre jeunesse, «Très Tôt Théâtre» aux Éditions Le Mot de passe. Il accueille ainsi dans un catalogue très coloré, aux livres en partie illustrés, les figures françaises déjà citées au côté desquelles on peut également trouver Claude Morand, quelques auteurs émergeants comme Karin Serres et des textes en traductions (Seul de Börje Lindström, traduction d'Anne Barlind, par exemple). La collection publiera jusque dans le milieu des années 1990, son fonds sera repris et diffusé par Théâtrales. Les années 1988 et 1989 voient également l'apparition de deux autres collections de théâtre jeunesse, qui correspondent à deux tendances éditoriales fondamentales pour le secteur : d'une part, à l'instar du TJA, le CDNEJ La Fontaine à Lille dirigé par René Pillot, qui confie à Jeannine Pillot la création des Éditions La Fontaine pour publier les textes créés au théâtre (dont le premier texte de Philippe Dorin, Villa Esseling Monde); d'autre part, l'animateur militant de l'éducation populaire et de l'action théâtrale Émile Lansman, crée les Éditions Lansman, non exclusivement consacrées à la publication du théâtre jeunesse, mais dont il est un élément central dans le catalogue.

À la fin des années 1980, nous sommes ainsi en présence de trois tendances éditoriales : la publication par une structure théâtrale; la collection «émancipée» du plateau; l'action culturelle en direction des compagnies et des animateurs. La quatrième tendance est celle d'un éditeur spécialisé en littérature jeunesse. En 1995, Brigitte Smajda, enseignante de français dans le secondaire et auteure de romans jeunesse, fonde la collection "Théâtre» au sein d'une maison prestigieuse, L'École des loisirs, qui va devenir en quelques années la collection en tête du marché, accueillant notamment les textes de Philippe Dorin, Nathalie Papin ou Karin Serres. Enfin, la dernière tendance forte du secteur est celle de la diversification en direction de la jeunesse des éditeurs spécialisés de théâtre contemporain, avec les créations successives de : "Heyoka jeunesse» en 1999; "Théâtrales Jeunesse» en 200I ; "L'Arche jeunesse» en 2005; plus tard, en 2009 "Théâtre Jeunesse» chez Espace 34; et récemment en 2014 "Jeunesse» aux Solitaires Intempestifs.

\section{Enjeux éditoriaux : les forces en présence et l'économie du secteur}

Aujourd'hui, le secteur de l'édition de théâtre jeunesse représente environ une cinquantaine de titres nouveaux chaque année, avec un rythme de publications 
d'environ 4 à Io titres par an et par éditeur. Les acteurs principaux déjà cités sont, classés par ordre chronologique de création :

- les Éditions La Fontaine (Lille), dirigées par Soazic Courbet (créées par Jeannine Pillot en 1989), une soixantaine de titres jeunesse au catalogue;

- les Éditions Lansman (Belgique), dirigées et créées par Émile Lansman en 1989, IIo titres au catalogue répartis dans plusieurs collections ${ }^{9}$;

- la collection "Théâtre» de L'École des loisirs (Paris), dirigée et créée par Brigitte Smajda en 1995, I35 titres publiés;

- la collection «Heyoka» chez Actes Sud-Papiers (Paris et Arles), dirigée et créée par Claire David et Dominique Bérody en 1999, 65 titres au catalogue; - la collection «Théâtrales Jeunesse» des Éditions Théâtrales (Montreuil), créée par Jean-Pierre Engelbach et Françoise du Chaxel en 200I, collection que je dirige avec cette dernière, 75 titres au catalogue;

- la collection «L'Arche Jeunesse» des Éditions de L'Arche (Paris), créée et dirigée par Rudolf Rach en 2002, 35 titres au catalogue;

- la collection "Théâtre Jeunesse» des Éditions Espace 34 (Les Matelles), créée et dirigée par Sabine Chevallier en 20I0, Io titres.

Dans ce bref tableau, il est nécessaire de mentionner également quelques titres publiés par d'autres éditeurs spécialisés en théâtre comme l'Avant-Scène Théâtre, Art et Comédie, ou Le Petit Bonhomme Vert, maison ayant mis fin à ses activités en 20I3, qui avait inventé le concept du "théâtre illustré», autrement nommé «l'album-théâtre ${ }^{\mathrm{IO}}$ »; mais également de maisons spécialisées en jeunesse ou en parascolaire qui publient quelques titres de théâtre comme Retz ou Syros.

En termes d'économie éditoriale, les tirages initiaux moyens de ces collections oscillent entre I 500 à 6000 exemplaires, ce qui les place au-delà des tirages initiaux connus dans les collections de théâtre contemporain généralistes (entre 800 et 2500 exemplaires selon les éditeurs). Les formats de ces ouvrages sont majoritairement de type poche, correspondant ainsi aux canons esthétiques de l'édition jeunesse. En termes d'auteurs, on constate que $30 \%$ des auteurs sont également publiés en théâtre généraliste avec des exemples forts comme Olivier Py ou Jean-Claude Grumberg chez Actes Sud-Papiers, Fabrice Melquiot

9. Les principales collections chez Lansman accueillant des titres jeunesse : «Urgence de la jeune parole»; "Lansman Jeunesse»; "Tous en scène»; "Théâtre pour la jeunesse WallonieBruxelles "; "Théâtre à lire et à jouer»; "Cahiers du Soleil debout»; "Printemps théâtral ».

IO. Je reprends là l'expression créée par $\mathrm{M}$. Bernanoce qui a consacré plusieurs articles à cette part du théâtre jeunesse. Voir par exemple «L'album-théâtre : typologie et questions posées à sa lecture", Modernités, $n^{\circ}$ 28, L'album contemporain pour la jeunesse. Nouvelles formes, nouveaux lecteurs?, C. Connan-Pintado, F. Gaïotti, B. Poulon (dir.)Bordeaux, Presses universitaires de Bordeaux, 2008, p. 39-52. 
à L'Arche, Sylvain Levey ou Stéphane Jaubertie chez Théâtrales. Par ailleurs, en comparaison avec les collections généralistes, la part des auteurs étrangers est légèrement moindre avec $15 \%$ de titres traduits contre près de $30 \%$, avec une surreprésentation des auteurs anglo-saxons (Mike Kenny, Daniel Keene...).

Ce secteur de niche connaît également des niveaux de vente très importants pour certains titres, semblables à quelques titres phares de l'édition jeunesse, férue de séries. On peut citer ainsi l'ouvrage le plus vendu en théâtre jeunesse de ces dix dernières années: Le Long Voyage du pingouin vers la jungle de JeanGabriel Nordman, publié en $200 \mathrm{I}$ et qui a dépassé les 80 o00 exemplaires vendus. D'autres opus atteignent également des chiffres élevés comme Le Petit Violon de Jean-Claude Grumberg, au-delà des 50 ooo exemplaires; ou L'Ogrelet de Suzanne Lebeau, au-delà des 30 ooo exemplaires. Si l'on ajoute que le secteur spécialisé de théâtre généraliste a connu depuis une dizaine d'années un bouleversement éditorial en termes de tirages, de ventes et de cessions du fait de l'arrivée de ces collections jeunesse, comment s'explique ce phénomène? Le facteur principal a été et reste encore aujourd'hui la prescription de l'école. En effet, en 2002, la Direction générale de l'Enseignement scolaire a instauré des «listes de références pour une première culture littéraire en cycle 3 ". Non programmatiques, mais incitatives, ces listes ont accueilli le genre théâtral au sein d'autres domaines comme le roman, l'album, la bande dessinée ou la poésie, notamment. Renouvelées en 2004, puis en 2007, année de la création d'une nouvelle liste de titres de théâtre en cycle 2 cette fois-ci, ces listes ont permis à ces éditeurs dont la surface médiatique demeure modeste et dotés de réseaux professionnels non tournés initialement vers cette prescription scolaire, de profiter des chiffres de vente que le seul public "classique» de théâtre ne peut offrir : la cible de l'école étant beaucoup plus importante. Certains éditeurs comme Théâtrales ont également cherché à amplifier ce phénomène en produisant des outils éditoriaux de repérage et de critique ${ }^{\mathrm{II}}$, ou des outils visant plus directement l'utilisation pédagogique de ces textes ${ }^{12}$. Cette prescription scolaire a ainsi favorisé le décollage de ces collections malgré l'absence de dimension programmatique. Récemment, en 20I4, de nouvelles listes incitatives ont été instaurées au collège, mais il est encore trop tôt pour analyser leurs conséquences.

II. M. Bernanoce a ainsi publié chez Théâtrales (en coédition avec le CRDP de Grenoble pour le premier tome) À la découverte de cent et une pièces (2006) et Vers un théatre contagieux (2012), les deux premiers volumes d'une entreprise de «Répertoire critique et analytique du théâtre contemporain pour la jeunesse».

I2. Les Carnets artistiques et pédagogiques des Éditions Théâtrales Jeunesse sont disponibles en ligne depuis 2009. 


\section{Quelles visées thématiques?}

Mon objectif étant d'établir un cadre économique et réticulaire de l'édition du théâtre jeunesse, je n’aborderai pas la dimension formelle défendue par ces éditeurs. Un mot simplement, pour avancer qu’à l'instar du théâtre généraliste le théâtre jeunesse publié connaît une diversité dramaturgique importante, sans doute plus radicale formellement que le généraliste, pour des raisons en partie d'économie théâtrale. Voici une rapide typologie formelle : en analysant les catalogues, on observe des tendances nettes, avec une représentation importante d'un théâtre-récit, d'un théâtre symboliste, d'un théâtre épistolaire ou diariste et d'un théâtre proche du matériau dramaturgique. Le point commun à ces écritures serait la persistance du drame, les auteurs visant sans doute des lecteurs jouissant de points de repères théoriques moins ancrés. Mais face à ce "vrai public» comme le défend Jean-Claude Lallias, quelles thématiques sont privilégiées par les éditeurs? On pourrait objecter que les auteurs sont à l'origine de ces thèmes par leur liberté d'écriture (parfois contrariée ou favorisée par les commandes), mais je vise bien ici le geste éditorial qui consiste en une médiation, donc à des choix. Sachant, comme évoqué plus avant, que l'enjeu économique de la prescription scolaire est primordial, les choix des éditeurs sont-ils encadrés par des tabous thématiques?

Cela ne semble pas être le cas, alors même que ces collections s'adressent au champ large des jeunes (à savoir à la fois les jeunes lecteurs, mais également leurs médiateurs, qu'ils soient les enseignants, les animateurs d'ateliers, comme tout le secteur professionnel du théâtre) et que l'édition jeunesse est encadrée par la loi de 1949 relative aux publications à destination des enfants et adolescents qui interdit l'édition de titres faisant l'apologie «du vol, de la paresse...». Or, en empruntant comme corpus représentatif l'ouvrage de Marie Bernanoce, $\grave{A}$ la découverte de cent et une pièces, on relève un certain nombre de thématiques présentes dans les iı6 pièces publiées analysées. Si les thèmes dominants sont, sans surprise, l'enfance (20 textes) ou le rêve (I3 textes), des thématiques plus dures et sombres sont très présentes : la mort (I5 textes), la guerre ( 13 textes), la violence (8 textes), le divorce (6 textes). Cette évolution semble «classique» en terre de littérature jeunesse, le théâtre ne faisant pas exception, même si les éditeurs, lorsqu'on analyse leur communication (que cela soit sur les sites internet ou dans les catalogues papier), mettent très peu en avant cette dimension purement thématique, ce qui tranche avec les confrères européens. 


\section{Une exception culturelle française? La situation en Europe}

Pour établir des points de comparaison avec la situation française, il est intéressant d'observer la situation dans trois pays différents : l'Angleterre, l'Allemagne et l'Espagne, car dans ces pays de forte tradition théâtrale le livre de théâtre, contrairement à la France, est principalement vécu comme un objet de médiation théâtrale visant à la recherche de production et accompagnant toujours celle-ci. Ainsi, la publication est en grande partie concomitante avec des créations scéniques, ce qui constitue une différence nette avec la situation française. L'autre façon de publier le texte de théâtre jeunesse en Europe serait de prendre appui sur une dimension sociale, voire sociétale, assignant aux auteurs une mission d'intérêt général. En effet, de nombreuses commandes aux auteurs sont passées par des institutions scolaires pour traiter de sujets. On parle ainsi en Angleterre des school plays sur des thématiques sociales comme le suicide chez les adolescents pour générer, à la suite de la pièce représentée, un débat. La démarche est socioculturelle et les éditeurs publient ces textes comme supports à celle-ci.

Quels sont les acteurs principaux? Comme en France, l'Angleterre connaît un secteur d'éditeurs spécialisés en théâtre dans lequel les collections dédiées aux jeunes sont présentes à hauteur d'environ 5 à Io \% du nombre de titres dans des catalogues assez dirigés vers le plateau comme en atteste la position d'agents qu'on retrouve de façon indissociable (comme en Allemagne). Prenons l'exemple de Nick Hern Books : «Theater publishers \& performing rights agents». Cet éditeur dont le catalogue compte près de mille titres contemporains accueille quatre-vingts titres for children dont une cinquantaine de versions de textes exclusivement scéniques (stage version) notamment de grands classiques de la littérature mondiale. Autre particularité anglaise, une présence numérique beaucoup plus forte qu'en France (les titres de théâtre jeunesse n'étant pour l'instant pas disponibles au format numérique chez les éditeurs français) avec des éditeurs faisant cohabiter les versions papier et numérique des textes de théâtre jeunesse, comme c'est le cas pour Heuer Publishing qui accueille des collections aux intitulés évocateurs : "Youth Theatre», "Community Theatre», «Holiday Theatre», "Ten Minute Plays», renforçant l'aspect utilitaire du geste éditorial. Enfin, les écoles et autres ateliers anglais ont également accès aux éditeurs dits "pure players» à savoir exclusivement présents en ligne, comme Plays for Kids qui annonce proposer : "A selection of drama scripts designed for school plays, youth theatre and anywhere else children and teenagers get to grips with plays ${ }^{13}{ }^{13}$, avec

I3. "Une sélection de scénarios dramatiques dédiés aux pièces d'école, au théâtre pour les jeunes, partout où les jeunes et les adolescents chercher à se familiariser avec le jeu » (je traduis). 
des catégories comme : «School Plays, Play with Education theme, Christmas Play, Reader's Theatre, Play with Historicals Subjects, Plays for Children with Religious, Moral Themes; Rhyming Plays", des thématiques très précises, fortement mises en avant dans la communication de ces éditeurs.

En Allemagne, les fameux Verlage défendent avant tout des auteurs qu'il s'agit de faire produire sur les scènes et force est de constater que l'impact de ces publications, qui deviennent des outils de promotion pour la recherche de créateurs scéniques et de producteurs, est beaucoup plus important et efficient que les publications françaises. Ainsi, Felix Bloch Erben se définit comme étant un "Verlag für Bühne, film und Kinder-Jugendtheater"14 et on trouve à son catalogue de nombreux auteurs allemands et autrichiens mais également quelques traductions comme les textes de l'Anglais Mike Kenny, dont les textes sont disponibles également en téléchargement. Chez Verlag der Autoren, les publications jeunesse représentent IO \% des publications annuelles, mais comptent un nombre beaucoup plus large de textes que dans les catalogues français avec près de trois cents textes jeunesse pour "petits et adolescents». De plus, les liens entre ces éditeurs et la quarantaine d'institutions dédiées à la jeunesse disséminées sur tout le territoire fédéral allemand sont très présents comme en atteste la communication de ces Verlage.

En Espagne, la démarche éditoriale correspond principalement à de l'action culturelle, puisque l'essentiel des pièces pour la jeunesse espagnoles sont publiées dans des anthologies que l'Assitej ${ }^{\text {is }}$ Espagne propose dans sa "Coleccion Teatro». À travers ces trois exemples, on observe que l'édition théâtrale jeunesse en France demeure assez éloignée d'une vision purement utilitariste du texte de théâtre et vise une tradition littéraire, comme le montre l'impact de la prescription scolaire sur les chiffres de ventes. Mais des évolutions récentes modifient la situation. Elles tendent à montrer que l'impact modeste de ces collections françaises sur la programmation des spectacles tendrait à se consolider, quitte, pour les éditeurs, à procéder à des choix plus directement en liens avec des structures théâtrales.

\section{Quel impact? Quelles évolutions récentes?}

Dans son ouvrage, Le théâtre jeune public, un nouveau répertoire (Presses universitaires de Rennes, 2009), Nicolas Faure montre qu'entre 1970 et 1990 les programmations jeune public laissaient une part minoritaire aux textes

I4. Je traduis : "édition théâtrale et cinématographique pour enfants».

I5. Association internationale du théâtre pour l'enfance et la jeunesse. 
avec $55 \%$ de textes issus de création collective, $24 \%$ d'adaptations et $19 \%$ de textes. Son étude contient des chiffres avant l'apparition des principales collections de théâtre contemporain jeunesse, et lorsqu'il propose des chiffres recueillis pour les années 2000, il annonce seulement Io à $15 \%$ de textes d'auteurs dans le spectacle de théâtre jeunesse, ce qui me paraît correspondre à une analyse plutôt partielle car ne permettant pas de mesurer l'impact réel des éditeurs. Aussi, après une quinzaine d'années de publications spécialisées, de quel impact justement le secteur peut-il se prévaloir ${ }^{16}$ ? Je dirai que cet impact demeure modeste par rapport au théâtre généraliste, mais que la part des textes dans les programmations s'affermit peu à peu : le rôle des éditeurs peut sans doute être mis en avant. Deux exemples : au festival "Théâtre à tout âge» organisé par Très Tôt Théâtre à Quimper, seulement $9 \%$ des spectacles proposés en 2012 étaient issus de textes publiés, contre I $8 \%$ en 2013 et I $6 \%$ en 20I4; au festival Méli'mômes de Reims, I $\%$ de textes publiés en $2012,17 \%$ en $2013,15 \%$ en 2014 . Et sur ces chiffres environ la moitié des textes avaient été publiés au moment des créations, ce qui montre une évolution inverse de l'émancipation de la publication par rapport à la programmation.

Est-ce à dire que l'engagement initial des éditeurs tend à se déliter quelque peu et que dans le même temps on ne monterait que les textes entrant dans les canons de la prescription scolaire? Je serais moins sévère avec le secteur qui demeure pour au moins un tiers de ses publications dans la volonté de se placer en amont de la programmation. De plus, les éditeurs ont contribué à poser un label sur bon nombre d'auteurs afin que ces derniers soient davantage repérés. Le glissement qui s'opère est celui d'un nombre très important de dispositifs en faveur des auteurs, comme des résidences ou des commandes de la part de compagnies ou de théâtre dédiés à la création jeunesse, ce qui implique que les publications sont davantage concomitantes aux créations, alors que les maisons ont contribué à les générer en assumant une politique de suivi des auteurs. Par ailleurs, le secteur jouit d'un embryon de légitimité littéraire car, au-delà de leur présence dans les programmes scolaires, les textes et les auteurs de théâtre jeunesse sont de plus en plus accueillis dans les salons du livre jeunesse dont celui de Montreuil. Et, en termes de légitimité théâtrale, les liens étant cycliques, la récente coédition entre la collection jeunesse de l'Arche avec le Théâtre AmStramGram de Genève dirigé par

I6. On est encore loin de l'impact et des liens de l'édition avec la programmation généraliste : le taux de création des textes contemporains publiés est passé de $59 \%$ des spectacles à $72 \%$ dans la sphère professionnelle et de $65 \%$ à $70 \%$ chez les amateurs. 
l'auteur Fabrice Melquiot n'est pas sans rappeler un illustre précédent entre cette maison et le TNP de Vilar.

Peut-être pourrait-on voir là le signe emblématique de l'inscription du secteur jeunesse dans une tradition revivifiée de théâtre "élitaire pour tous", pour reprendre la formule d'Antoine Vitez, et fortement inscrit dans une démarche de transmission. 